\title{
Chondrosarcoma of the Chest Wall: A Review of 53 Cases from Two Institutions
}

\author{
ULRICH LENZE $^{1}$, ANDREA ANGELINI ${ }^{2}$, FLORIAN POHLIG ${ }^{1}$, CAROLIN KNEBEL $^{1}$, \\ GIULIA TROVARELLI ${ }^{2}$, ANTONIO BERIZZI ${ }^{2}$, ANDREAS F. MAVROGENIS ${ }^{2}$, \\ JOERG THEISEN ${ }^{3}$, RUEDIGER VON EISENHART-ROTHE ${ }^{1}$ and PIETRO RUGGIERI $^{2}$ \\ ${ }^{1}$ Department of Orthopedics, Klinikum rechts der Isar, Technical University, Munich, Germany; \\ ${ }^{2}$ Department of Orthopedics and Orthopedic Oncology, University of Padova, Padua, Italy; \\ ${ }^{3}$ Department of Surgery, Klinikum rechts der Isar, Technical University, Munich, Germany
}

\begin{abstract}
Background/Aim: Chondrosarcomas (CS) of the chest wall are rare, but present an aggressive biological behavior compared to CS of the extremities. The aims of the present study were to determine factors associated with oncological outcomes as well as complications. Patients and Methods: We retrospectively analyzed 53 patients (42 primary, 11 recurrent tumors). In total, 39 central CS, 10 peripheral CS, 3 dedifferentiated CS and 1 mesenchymal CS were included. The ribs were most commonly affected (68\%). Overall survival and disease-free survival were estimated with Kaplan-Meier analyses and compared with log-rank test. Results: Mean follow-up was 7 years. Negative margins were achieved in $87 \%$ of patients. Thirty patients (57\%) remained continuously disease-free (NED), three (5\%) NED after treatment of relapse, seven $(13 \%)$ were alive with disease, twelve (23\%) were dead with disease and one of other cause. The 10-year survival rate was $81 \%$ and $45 \%$ in primary and recurrent tumors, respectively. Survival was significantly affected by tumor stage $(p<0.001)$, local recurrence $(p=0.025)$ and metastases $(p=0.002)$. Six complications (16\%) were observed. Conclusion: The outcome is rather poor, especially in patients with local recurrence. Presumably due to a high biological aggressiveness, a stricter definition of surgical margins should be considered for this location.
\end{abstract}

Chondrosarcomas account for about $20 \%$ of all primary malignant osseous tumors, representing the third most

Correspondence to: Pietro Ruggieri, MD, Ph.D., Department of Orthopedics and Orthopedic Oncology, University of Padova, Via Giustiniani 3, Padua, Italy. Tel: +39 0498213311, Fax: +39 0498213365, e-mail: pietro.ruggieri@unipd.it

Key Words: Sarcoma, chondrosarcoma, chest wall, bone tumor, sternum. common primary malignancy of bone after myeloma and osteosarcoma $(1,2)$. They are most frequently located in the pelvis (ilium), followed by proximal femur, proximal humerus and distal femur $(3,4)$. The chest wall (primarily the ribs) is affected in about $15 \%$ of cases, and thus the annual incidence is extremely low for this manifestation site $(5,6)$. However, chondrosarcoma represents the most common primary bone tumor involving the ribs and sternum (7). Tumor-related factors, such as tumor grade and localization, have been shown to be independent predictors of chondrosarcoma patient survival (3). The rate of metastases has been also found higher in pelvic and chest wall chondrosarcomas, compared to conventional chondrosarcomas of the extremities (8). Thus, involvement of pelvis and chest wall have been associated with a poorer outcome. Particular characteristics of chest wall chondrosarcoma, like its close proximity to vital organs, its indolent nature, and the high risk of local recurrence have been further emphasized $(5,8,9)$.

Published studies on the outcome and prognostic factors of the patients with chondrosarcomas of the chest wall are relatively limited (5-15). Therefore, we performed this study to evaluate the distribution patterns, natural history and tumor-related data of patients with chondrosarcoma of the chest wall, and analyze patients' outcome with respect to survival and complications after surgical treatment.

\section{Patients and Methods}

We retrospectively studied all patients with histologically documented chondrosarcomas of the chest wall (sternum, clavicle, ribs) that were treated between 1981 and 2015 at two tertiary tumor centers. The criteria of patient selection were: 1) confirmed histological diagnosis of chondrosarcoma of the chest wall; 2) index surgical treatment not aiming to palliation, thereby excluding patients treated only with palliative surgery, chemotherapy and/or radiotherapy; and 3) minimum follow-up of two years.

Imaging studies and histological sections for all patients were rereviewed for the purpose of this study. Information regarding the 
clinical characteristics, treatment and outcome were collected. Imaging studies used to define the extension of the primary tumor included radiography, bone scintigraphy, computed tomography (CT) and magnetic resonance imaging (MRI) (some methods varied with time and, hence, availability). Chest CT scan was also available after 1991. All patients or their relatives gave written informed consent for their data to be included in this study; an Institutional Review Board/Ethics Committee approval is not necessaery for retrospective studies at the authors' institutions.

Patient-related data (age and gender), tumor characteristics (site of involvement, histology, volume and extension, stage and grading, and presence of metastases at diagnosis), and treatment-related data (previous surgical treatment, type of local treatment, type of reconstruction, and surgical margins) were evaluated and further analyzed for possible correlations with patients' outcome. Tumor volume was measured on the resected specimen; the maximum height, width, and depth were recorded and the volume was calculated using the formula of an ellipsoid mass volume $=[(\pi / 6) \times$ height $\times$ width $\times$ depth]. We staged patients using the Musculoskeletal tumor society surgical staging system, which is the system routinely used at our institutions. The resection margins were classified according to Enneking in: wide, marginal and intralesional resections. Complications were subdivided in postoperative (within one month after surgery), early (between one and six months after surgery) and late (more than six months after surgery) complications.

Routine follow-up evaluation was performed every 3 months for the first 2 years, every 6 months for the next 3 years, and then annually. Each follow-up evaluation included clinical evaluation and imaging studies with standard radiographs, CT scan, and MRI; a CT scan of the chest was performed every 6 months for the first 2 years and then annually. The patients were distributed into four groups according to their clinical status at last follow-up: 1) continuously disease-free (no evidence of disease NED); 2) disease-free after treatment of local recurrence or metastasis; 3 ) alive with disease (AWD), presenting local recurrence or metastasis; and 4) dead with disease (DWD) or dead of other disease (DOD), patients who died from the tumor or other unrelated causes, respectively. Survival was defined as the time interval from the date of diagnosis to the date of last follow-up or the date of death from any cause. Disease-free survival (DFS) was defined as the time interval from the date of diagnosis to the date of the first event (recurrent or progressive disease, and death from any cause) or the date of last follow-up for patients who had no events.

Survival and DFS distributions were estimated using a the Kaplan-Meier survival analysis; prognostic factors and their influence on survival were determined with the log-rank test. The level of significance was set to 0.05 . The effect level of clinical characteristics on survivorship was evaluated using both the univariate Kaplan-Meier analysis and the multivariate Cox regression analysis with stepwise forward selection. The data were recorded in Microsoft Excel ${ }^{\circledR} 2003$ spreadsheet (Microsoft Inc, Redmond, WA) and analyzed using MedCalc ${ }^{\circledR}$ Software Version 11.1 (MedCalc Software, Mariakerke, Belgium) and SPSS 25.0 (IBM, Armonk, NY, USA).

\section{Results}

Fifty-three consecutive patients with chondrosarcomas of the chest wall were finally included in the study. Mean follow-up was 6.9 years (median: 4.9 years, range $=2-17$ years), the predominant gender was male [38 males (72\%) vs. 15 females
Table I. Histological types of the CS of the patients included in this series.

\begin{tabular}{lcc}
\hline CS type & N & Frequency \\
\hline Central conventional & 39 & $73.6 \%$ \\
Secondary peripheral & $10^{*}$ & $18.9 \%$ \\
Dedifferentiated & 3 & $5.7 \%$ \\
Mesenchymal & 1 & $1.9 \%$ \\
\hline
\end{tabular}

CS: Chondrosarcoma. *One patient with multiple osteochondromas.

$(18 \%)$ ], and mean age at diagnosis was 49 years (range $=11$ 87 years). In total, three patients (6\%) were younger than 20 years, $14(26 \%)$ were between 20 and 40 years, $21(40 \%)$ between 41 and 60 years, and 15 patients (28\%) were older than 60 years. The most common tumor location was ribs (36 patients, $68 \%$ ), followed by sternum (14 patients, $26 \%$ ) and clavicle (3 patients, $6 \%$ ), while the most common histology was central conventional chondrosarcoma (Table I). The tumor volume ranged from $6.24 \mathrm{~cm}^{3}$ to $2,240 \mathrm{~cm}^{3}$. The initial tumor stage at presentation was stage Ib in 12 patients (23\%), stage IIb in $36(68 \%)$ and stage III in five patients (9\%). Of the latter patients, four had pulmonary and one patient had lymph node metastases (Figure 1). Eleven patients $(21 \%)$ were surgically treated elsewhere, and were subsequently referred to our institution with a recurrent/persistent tumor. Of these, one patient with costal chondrosarcoma was not fit for surgery and received palliative radiotherapy. In total, 52 tumor resections were performed in our institutions (Table II).

Thirty-five patients with costal chondrosarcomas were offered surgical treatment, while an extended tumor resection was performed in eight cases: up to sternum in three patients; to vertebrae in two patients; to lungs in two patients; and to the abdomen in one patient. The decision upon reconstruction of the bony defect, was based mostly on the extent of the resection and the consequences it might have on the dynamic compliance of the thoracic wall. Thus, a reconstruction was performed in defects greater than $4 \mathrm{~cm}$. For the bony defect reconstruction, either a bridging plate or a Kirschner-wire osteosynthesis was performed in 14 patients (40\%; in 13 patients with one or two plates, in 1 patient with a Kirschner-wire). In 21 patients (60\%) no reconstruction of the bony defect was conducted. Soft tissue reconstruction was performed in 27 patients (76\%): in 18 patients using a mesh [polytetrafluoroethylene (Gore-Tex) or high-density polyethylene (Marlex)], while two of them received an additional polymethylmethacrylate augmentation, and three a muscle flap (latissimus dorsi muscle and pectoralis major muscle); in nine patients an autologous fascia lata transplant was utilized. In the remaining eight patients (23\%) no soft tissue reconstruction was performed.

Fourteen patients with chondrosarcoma of the sternum were treated with partial sternectomy (defined as resection of $<90 \%$ 

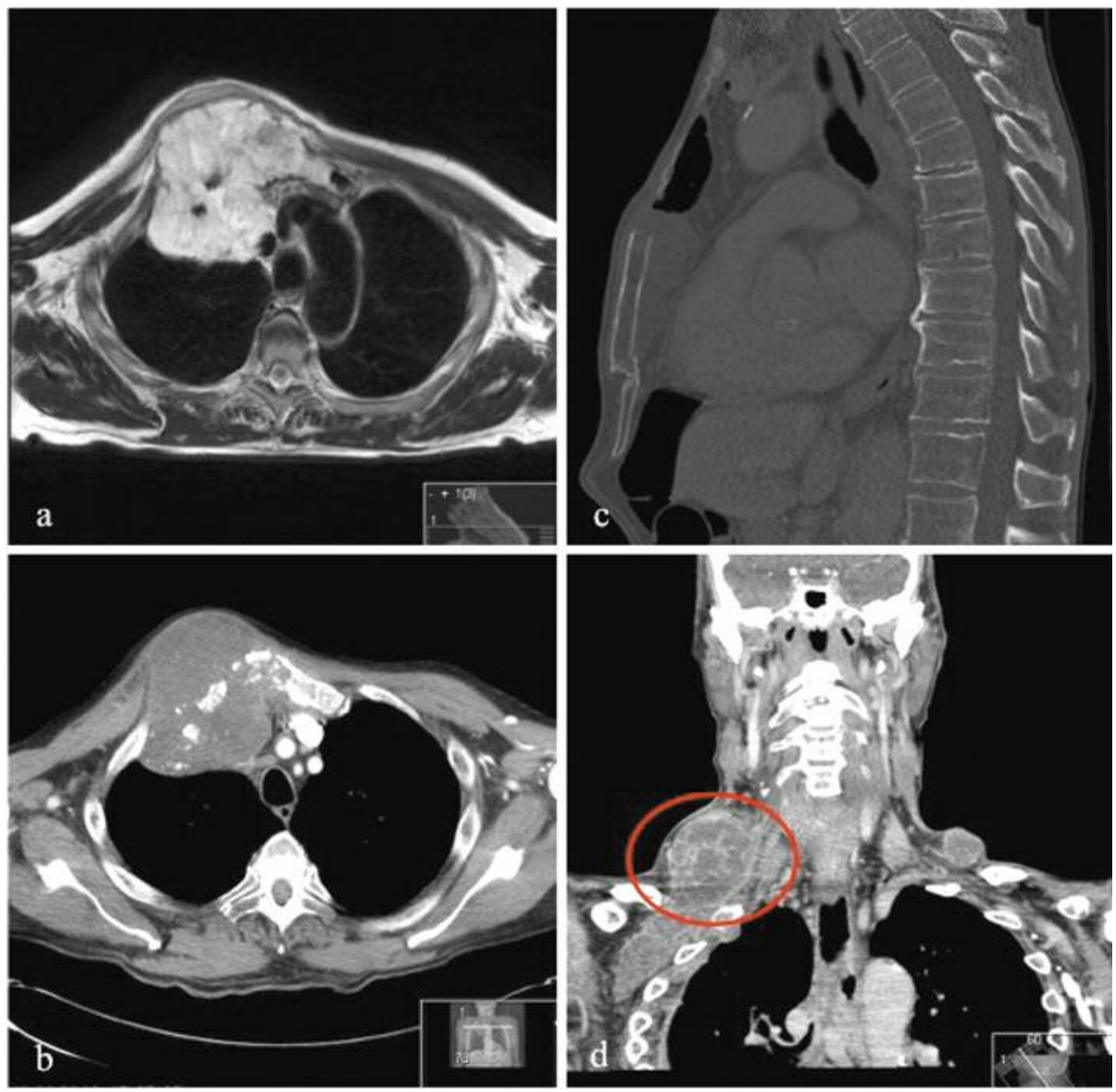

Figure 1. Chondrosarcoma of the right 3rd to 5th rib (grade 2, stage III) in an 87-year-old patient with protrusion into the thoracic cavity (a: axial T2-weighted MRI, $b$ : axial CT sequence). The patient underwent wide resection including partial sternectomy and resection of lung tissue ( $c$ : coronal $C T$ sequence). After 5 months, the patient presented with a local recurrence at the level of the superior thoracic aperture (d: coronal CT sequence).
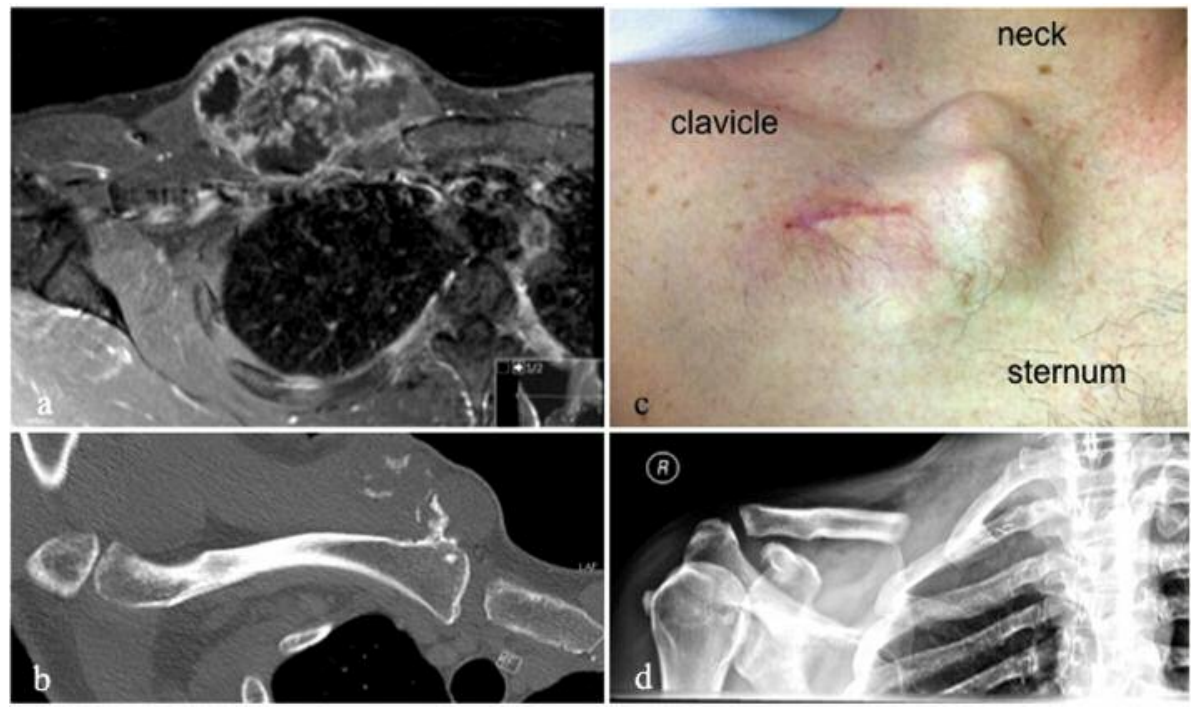

Figure 2. Peripheral chondrosarcoma of the right medial clavicle (grade 2, stage IIb) in a 44-year-old patient (a: T1-weighted MRI sequence with contrast agent, $b$ : axial CT sequence, $c$ : clinical photo of the tumor with biopsy scar). A wide resection was performed including the external pleura which was covered using a polytetrafluoroethylene mesh. The resected medial clavicle was not reconstructed in this case (d: postero-anterior radiograph). 
Table II. Type of treatment and reconstruction based on tumor location.

\begin{tabular}{|c|c|c|c|}
\hline Type of treatment & Patients (no.) & Bone reconstruction & Soft tissue reconstruction \\
\hline Thoracic ribs & $36(68 \%)$ & $14 / 35$ & $27 / 35$ \\
\hline Resection & 35 & $\begin{array}{l}\text { Bridging osteosynthesis with one or } \\
\text { two plates (13 patients) and with a } \\
\text { Kirschner-wire (one patient) }\end{array}$ & $\begin{array}{l}\text { Mesh* (18 patients) and autologous } \\
\text { fascia lata (9 patients) }\end{array}$ \\
\hline Limited to the ribs & 27 & & \\
\hline Extended to the sternum & 3 & & \\
\hline Extended to the vertebrae & 2 & & \\
\hline Extended to the lung & 2 & & \\
\hline Extended to the abdomen & 1 & & \\
\hline Palliative radiotherapy & 1 & & \\
\hline Sternum & $14(26 \%)$ & $10 / 14$ & $12 / 14$ \\
\hline Resection & 14 & $\begin{array}{l}\text { Bridging osteosynthesis with one or } \\
\text { two plates (10 patients) }\end{array}$ & $\begin{array}{c}\text { Mesh* (6 patients), Mesh and } \\
\text { muscle flap (1 patient), autologous } \\
\text { fascia lata (5 patients) }\end{array}$ \\
\hline Limited to the sternum & 7 & & \\
\hline Extended to the ribs & 5 & & \\
\hline Extended to the ribs and clavicle & 1 & & \\
\hline Extended to the diaphragm & 1 & & \\
\hline Clavicle & $3(6 \%)$ & $1 / 3$ & $1 / 3$ \\
\hline Resection & 3 & Autologous non-vascularized fibula & Mesh* and muscle flap \\
\hline
\end{tabular}

*High-density polyethylene (Marlex) or polyglactin (Vicryl).

of the sternum in longitudinal diameter). Resections were extended to the ribs in five patients, the ribs and clavicles in one patient, and the diaphragm in one patient. The bony chest wall defect was reconstructed in ten patients $(71 \%)$ using bridging osteosynthesis plates. In the remaining four, no reconstruction was required. Additionally, in seven patients (50\%) a mesh [high density polyethylene (Marlex) or polyglactin (Vicryl)] was used for soft tissue reconstruction and one of them received an additional muscle flap (pectoralis major muscle). In five patients an autologous fascia lata transplant was applied, while no further reconstruction was required in two patients.

After wide resection of all three clavicular chondrosarcomas, the bony defect was reconstructed only in one patient using a free non-vascularised fibula graft (Figure 2). In another patient, in whom the external pleura had to be resected, a mesh (polytetrafluoroethylene (Gore-Tex) was used for the soft tissue reconstruction. Due to the volume and localization of individual tumors, a wide resection was not attempted in all cases. Of the 52 patients in whom a surgery was performed, a wide resection was aimed in 47 patients $(90 \%)$. Of those, clear margins were achieved in 41 patients $(87 \%)$ and wide but contaminated margins in six patients (13\%). A marginal resection was aimed in five patients $(10 \%)$. In total six surgeryassociated complications were recorded in six patients $(12 \%)$. Postoperative complications (within a month) occurred in three patients $(6 \%)$ : one patient required intensive medical care with long-term ventilation ( 3 weeks), due to cardiopulmonary insufficiency; one patient deceased one week postoperatively
Table III. Outcome and characteristics of patients who developed local recurrences or metastases.

\begin{tabular}{lcc}
\hline & $\begin{array}{c}\text { Local recurrences } \\
12 / 52(23 \%)\end{array}$ & $\begin{array}{c}\text { Metastases } \\
15 / 52(28 \%)\end{array}$ \\
\hline Type of presentation & & \\
Primary group & $7 / 42(17 \%)$ & $2 / 42(5 \%)$ \\
Recurrent tumor group & $5 / 11(45 \%)$ & $3 / 11(28 \%)$ \\
Margins & & \\
Wide margins & $6 / 41(15 \%)$ & $8 / 41(20 \%)$ \\
Wide but contaminated margins & $6 / 6(100 \%)$ & $4 / 6(67 \%)$ \\
Marginal margins & $-/ 5(-\%)$ & $3 / 5(60 \%)$ \\
Stage at presentation & & \\
IIB & 12 & 10 \\
III & & 5 \\
Negative prognostic factors & & \\
on survival to relapse & & n.s. \\
Grade 3 & 0.004 & n.s. \\
Previous surgery & 0.024 & n.s. \\
Margins & n.s. & n.s. \\
Stage 3 & n.s. & 0.006 \\
Site & n.s. & \\
Age $>60$ years & n.s. & \\
\hline
\end{tabular}

n.s.: Not statistically significant.

after acute renal failure; and in one patient a revision surgery was performed 18 days after the initial procedure for resection of recurrent nodules on the pericardium. Two early complications (4\%) were recorded: one patient suffered from 


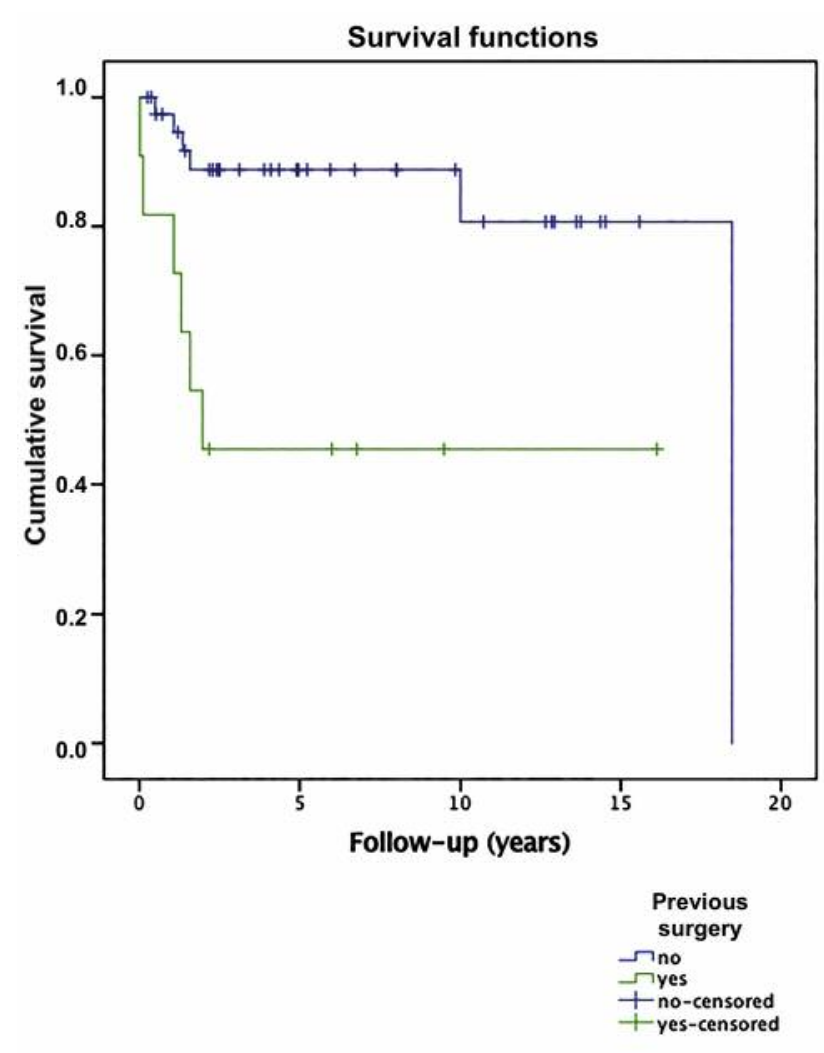

Figure 3. Kaplan-Meier curves show the overall survival of patients who presented at our Institutions with a primary chondrosarcoma (no previous surgery) and patients with a recurrent tumor (previous surgery). A statistical significant difference in the overall survival of the two groups was observed $(p=0.002)$.

pneumonia three months after tumor resection, and one patient presented wound dehiscence four months after index procedure. Lastly, one patient developed decubitus with a skin fistula 11 months after surgery (late complication), due to a prominent osteosynthesis plate.

At the last follow-up, 30 patients (56.6\%) were continuously disease-free, three $(5.7 \%)$ were disease-free after treatment of local recurrence, and seven (13.2\%) were alive with disease. Twelve patients $(22.6 \%)$ were dead with disease and one $(1.9 \%)$ was dead of other disease. Survival in the group of patients presenting with tumor for the first time was $89 \%$ after 5 years and $81 \%$ after 10 years, while survival in the group of patients with recurrent tumors was only $45 \%$ after 5 and 10 years (Figure 3 ). Univariate and multivariate analysis managed to show that the following factors were associated with worse survival: tumor stage III $(p<0.001$; Figure 4$)$, local recurrence ( $p=0.025$; Figure 5$)$, metastases during the course of disease $(p=0.002)$, and initial presentation with a recurrent tumor ( $p=0.002$; Figure 3$)$. Gender, age, localization, histology, grading, tumor size and achieved margins had no significant influence on survival.

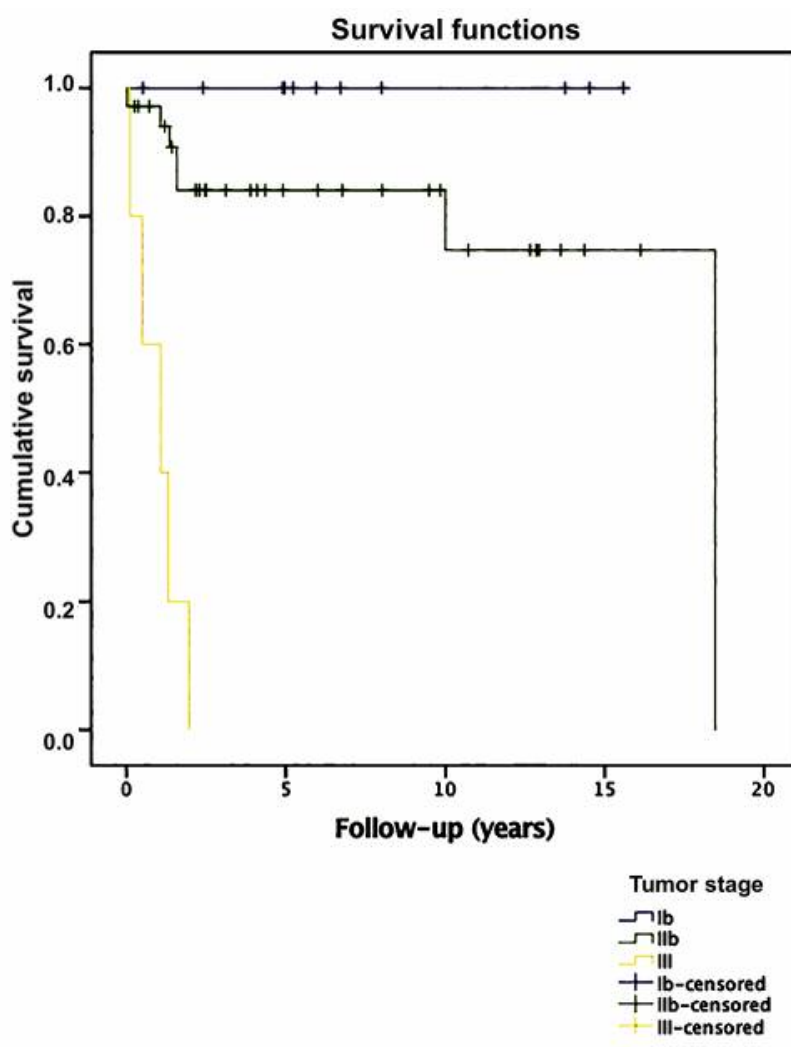

Figure 4. Kaplan-Meier curves show the overall survival of patients with tumor stage Ib, IIb and III. The tumor stage was a statistically significant influencing factor for the overall survival of patients with chondrosarcoma of the chest wall $(p<0.001)$.

Twelve out of 52 patients (23\%), who underwent surgical resection at our Institutions, developed local recurrences (Table III) after a mean period of 2.6 years (range=0.5-15.6 years). In 15 patients (28\%) metastases were recorded: 5 patients $(9 \%)$ with stage III tumors had metastases at presentation and 10 patients (19\%) with stage IIb tumors developed metastases during follow-up. In total 8 patients with metastases had died of disease at the latest follow up, 6 were alive with disease and one patient was alive with no evidence of disease after wedge resection of a pulmonary metastasis.

\section{Discussion}

Primary malignant tumors of the chest wall are rare (only about $1 \%$ of all primary tumors), but chondrosarcomas represent the most frequent histological type $(6,7,10-12)$. The ribs are thought to be more frequently affected than the sternum $(6,7,11,13-15)$. Likewise, in our study 36 patients (68\%) had costal chondrosarcoma, 13 patients $(24.5 \%)$ chondrosarcoma of the sternum and only four patients 


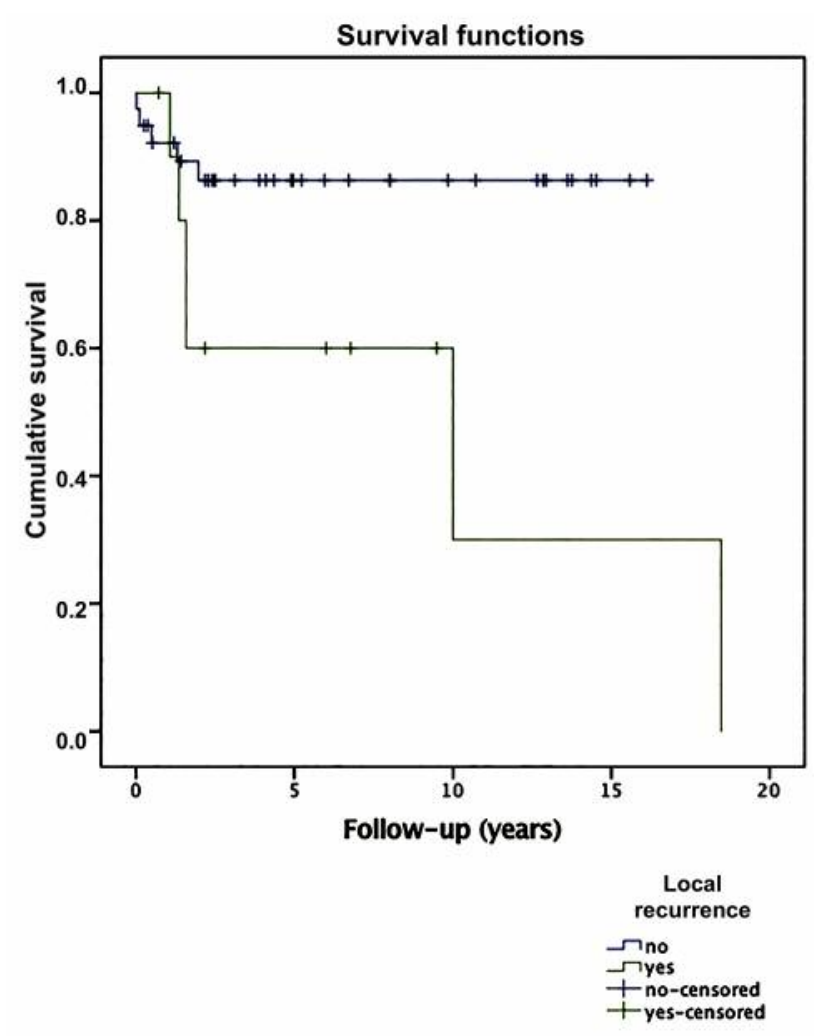

Figure 5. Kaplan-Meier curves show the overall survival of patients with and without local recurrence. The survival in patients with local recurrence was significantly worse $(p=0.002)$ compared to patients without local recurrence.

(7.5\%) had clavicular chondrosarcoma. Additionally, chondrosarcomas of the chest wall are most commonly seen anteriorly $(6,9)$. This was also confirmed in our series, where - besides sternal and clavicular chondrosarcoma - the majority of tumors of the ribs arose from the anterior chest wall and the costal cartilage $(78 \%)$. Chondrosarcomas of the clavicle were excluded in some publications on chest wall tumors, as the clavicle is not a flat bone (other than the sternum and the ribs) and not an intrinsic part of the thoracic cage (8). However, one can sometimes hardly differentiate whether the tumor originates from the sternum or the sternal part of the clavicle. Thus, the majority of included chondrosarcomas of the clavicle occurred in the medial part rather than the middle or lateral part of the clavicle. We have the opinion that chondrosarcomas of the sternum, ribs and clavicle show a similar biological behavior, differing from that of chondrosarcomas of the extremities, such as the shoulder or pelvis. Some studies reported that a pelvic CS is associated with poorer prognosis compared to appendicular tumors $(16,17)$. The aggressiveness of pelvic and chest wall CS is also demonstrated by the higher incidence of distant metastases compared to conventional CS of the extremities
(8). Our findings with respect to age are similar to chondrosarcomas of the extremities, where older age groups are more frequently affected than younger patients $(6,9,10$, $15)$. In our series $68 \%$ of patients were older than 40 years, and $28 \%$ above the age of 60 .

Despite several novel therapeutic approaches, surgery is still undisputedly the cornerstone in the treatment of chondrosarcoma, as chemotherapy and radiation therapy are not effective, due to the poor vascularity, extracellular matrix, and the low percentage of dividing cells $(3,16,18)$. In other histotypes that could affect the thoracic cage, such as breast cancer, radiation therapy represents a significant local adjuvant after radical surgery (19). This naturally applies to chondrosarcomas of the chest wall, which are, nevertheless, frequently found in close proximity to great vessels, as well as vital organs, such as the heart and lungs $(7,20)$. Surgical removal of the tumor usually leaves a large bone defect, with or without soft tissue defect, necessitating reconstruction in the same procedure $(15,20-22)$. Obviously, the need of a surgical approach for tumor resection should be considered a bias about the more frequent reconstruction of the thoracic cage compared to trauma. In fact, patients with serial rib fracture and blunt thoracic traumas (with or without flail chest) were usually non-surgically treated (23). One of the most controversially discussed issues in chondrosarcomas is the importance of clear surgical margins. The quality of margins, although it has not been shown to significantly influence overall survival in chondrosarcomas $(3,4,24,25)$, may influence the local recurrence rate (10). On the other hand, Marulli et al. (26), analyzed 89 chondrosarcomas of the chest wall, reporting that negative surgical margins, grade 1 tumors, age $\leq 55$ years and diameter $\leq 6 \mathrm{~cm}$ were independent predictors of better survival, whereas recurrence rate was influenced only by histological grade. In our series, the achieved margins neither significantly influenced survival nor local recurrence. In fact, half the patients with local recurrence had clear resection margins, and five of them developed also distant metastases. Additionally, seven patients from the primary tumor group developed tumor recurrence, despite the clear margins that have been achieved.

Results of this study showed that patients with recurrent tumors at presentation are more likely to develop another recurrence, and tumor recurrence was also associated with worse oncologic outcome $(p=0.025)$; this is in line with previous studies on prognostic factors in chondrosarcoma (24, $25,27)$. To our knowledge, there is only one study (28) in which the results in patients with primary and recurrent tumors of the chest wall were comparable. The collective in this study, however, was rather heterogeneous and also included entities such as Osteosarcomas, Ewing's sarcomas and even desmoid tumors. Some authors hypothesized, that the biological behavior of local recurrences might differ individually (3). Thus, a local recurrence following adequate treatment (clear 
margins) might be an expression of high biological aggressiveness, explaining the significant risk of these patients for developing metastases (3). Genetic and molecular aspects have been associated with biological aggressiveness of undifferentiated pleomorphic sarcomas (29). The biological aggressiveness of chest wall chondrosarcoma could be additionally emphasized by the rate of patients presenting metastases at time of diagnosis. In our cohort $5 \%$ of patients (with primary tumors) presented with metastases, which is slightly lower, as compared to the results of the Mayo Clinic and the Memorial Sloan-Kettering Cancer Center (6\% and $10 \%$, respectively) $(6,13)$. In comparable studies, with chondrosarcomas of the extremities and pelvis mainly, the reported metastatic rates at presentation were slightly lower being about $4 \%$ (17). Although not directly related, local progression of chest wall chondrosarcoma may additionally impair survival by causing respiratory and/or cardio-vascular insufficiency (8). In our cohort, four patients, who developed local recurrence, deceased from their disease without having metastases. This relation, along with the fact that high amount of patients develop local recurrence and/or metastases, despite clear margins, might promote the requirement of an adapted classification of margins at the chest wall. The Scandinavian Sarcoma Group proposed a stricter definition of margins for chondrosarcomas of the chest wall (8). The local recurrence rate in their analysis was highly dependent on the achieved margins; $4 \%$ after wide resections and $73 \%$ after intralesional resection. Wide surgical margins were defined by authors as intact pleura internally, intact muscle fascia externally, and transverse rib resection greater than $2 \mathrm{~cm}$ on both sides from tumor. Marginal surgical margins were assumed in cases, which were macroscopically and microscopically tumor-free, but within $2 \mathrm{~cm}$ distance from tumor. Tumors taken out in piecemeal technique or had microscopically positive margins, were classified as intralesional resections (8).

We acknowledge three limitations in this study. First, Type II statistical errors may exist for some of the end-points we considered. However, we believe that the long-term followup increases the power of our analysis, and allows us to assess prognostic factors on survival at the long term. Second, this study is limited by heterogeneity among groups (primary and recurrent tumors), and relative small numbers in some of our patient subgroups. However, the rarity and nature of the disease does not allow for large, blinded studies with equally distributed patient samples, even in our tumor referral centers. Additionally, in contrast to the present case series, primary malignant sarcomas of the chest wall are generally reported in small series that include lesions arising both in bone and cartilage. Of course, a larger sample size might help to draw important and meaningful conclusions regarding variables examined for this uncommon tumor location. Third, patients were treated in two musculoskeletal tumor centers, in Germany and Italy, with different surgical and reconstruction techniques. Nevertheless, as both centers are tertiary tumor centers with a long tradition in oncological surgery, a comparable and accurate surgical treatment can be assumed, despite different reconstruction techniques in some cases. Notwithstanding the aforementioned limitations, we report a relatively large cohort of patients with chest wall chondrosarcomas, and to our opinion, interesting conclusions can be drawn regarding this rare entity.

\section{Conclusion}

Chondrosarcomas of the chest wall are rare and most frequently affect male patients. Though the quality of margins was not a significant influencing factor for overall survival but only for local recurrence, a proper surgical treatment is of outmost importance in these patients. A high biological aggressiveness can be assumed due to the relevant number of patients with local recurrence despite clear margins at initial resection. The outcome is rather poor especially in patients with stage 3 tumors and patients with local recurrence. A localization-associated (close proximity to major vessels, the heart and the lungs) shortcoming is furthermore the fact, that local recurrence or progression might be life-threatening even without the presence of metastases.

\section{Conflicts of Interest}

There are no conflicts of interest to declare.

\section{Authors' Contributions}

The following Authors have designed the study (UL, AA, PR), gathered the data (UL, AA, FP, CK, GT), analyzed the data (UL, AA, AB), wrote the initial drafts (UL, AA, AFM), and ensure the accuracy of the data and analysis (UL, AA, JT, RvER, PR).

\section{References}

1 Limaiem F and Sticco KL: Cancer, Chondrosarcoma. [Updated 2019 Nov 12]. In: StatPearls. Treasure Island (FL): StatPearls Publishing; 2019. Available at: https://www.ncbi.nlm.nih.gov/ books/NBK538132/

2 Thorkildsen J, Taksdal I, Bjerkehagen B, Haugland HK, Borge Johannesen T, Viset T, Norum OJ, Bruland O and Zaikova O: Chondrosarcoma in Norway 1990-2013; an epidemiological and prognostic observational study of a complete national cohort. Acta Oncol 58(3): 273-282, 2019. PMID: 30632866. DOI: 10.1080/0284186X.2018.1554260

3 Andreou D, Ruppin S, Fehlberg S, Pink D, Werner M and Tunn PU: Survival and prognostic factors in chondrosarcoma: results in 115 patients with long-term follow-up. Acta Orthop 82(6): 749-755, 2011. PMID: 22066552. DOI: 10.3109/17453674. 2011.636668

4 Mavrogenis AF, Pala E, Angelini A, Ferraro A and Ruggieri P: Proximal tibial resections and reconstructions: clinical outcome 
of 225 patients. J Surg Oncol 107(4): 335-342, 2013. PMID: 22806768. DOI: $10.1002 /$ jso. 23216

5 Burt M, Fulton M, Wessner-Dunlap S, Karpeh M, Huvos AG, Bains MS, Martini N, McCormack PM, Rusch VW and Ginsberg RJ: Primary bony and cartilaginous sarcomas of chest wall: results of therapy. Ann Thorac Surg 54(2): 226-232, 1992. PMID: 1637209. DOI: 10.1016/0003-4975(92)91374-i

6 Rascoe PA, Reznik SI and Smythe WR: Chondrosarcoma of the thorax. Sarcoma 2011: 342879, 2011. PMID: 21647360. DOI: $10.1155 / 2011 / 342879$

7 Gonfiotti A, Santini PF, Campanacci D, Innocenti M, Ferrarello S, Caldarella A and Janni A: Malignant primary chest-wall tumours: techniques of reconstruction and survival. Eur J Cardiothorac Surg 38(1): 39-45, 2010. PMID: 20185323. DOI: 10.1016/j.ejcts.2009.12.046

8 Widhe B, Bauer HC and Scandinavian Sarcoma G: Surgical treatment is decisive for outcome in chondrosarcoma of the chest wall: a population-based Scandinavian Sarcoma Group study of 106 patients. J Thorac Cardiovasc Surg 137(3): 610-614, 2009. PMID: 19258076. DOI: 10.1016/j.jtcvs.2008.07.024

9 McAfee MK, Pairolero PC, Bergstralh EJ, Piehler JM, Unni KK, McLeod RA, Bernatz PE and Payne WS: Chondrosarcoma of the chest wall: factors affecting survival. Ann Thorac Surg 40(6): 535541, 1985. PMID: 2416278. DOI: 10.1016/s0003-4975(10)60344-X

10 Abdel Rahman ARM, Rahouma M, Gaafar R, Bahaa S, Loay I, Kamel M, Abdelbaki $\mathrm{H}$ and Yahia M: Contributing factors to the outcome of primary malignant chest wall tumors. J Thorac Dis 9(12): 5184-5193, 2017. PMID: 29312725. DOI: 10.21037/ jtd 2017.11 .61

11 Ito $\mathrm{T}$, Suzuki $\mathrm{H}$ and Yoshino $\mathrm{I}$ : Mini review: surgical management of primary chest wall tumors. Gen Thorac Cardiovasc Surg 64(12): 707-714, 2016. PMID: 27778223. DOI: 10.1007/s11748-016-0719-z

12 Shah AA and D'Amico TA: Primary chest wall tumors. J Am Coll Surg 210(3): 360-366, 2010. PMID: 20193901. DOI: 10.1016/j.jamcollsurg.2009.11.012

13 Burt M: Primary malignant tumors of the chest wall. The Memorial Sloan-Kettering Cancer Center experience. Chest Surg Clin N Am 4(1): 137-154, 1994. PMID: 8055278.

14 Fong YC, Pairolero PC, Sim FH, Cha SS, Blanchard CL and Scully SP: Chondrosarcoma of the chest wall: a retrospective clinical analysis. Clin Orthop Relat Res 427: 184-189, 2004. PMID: 15552156. DOI: 10.1097/01.blo.0000136834.02449.e4

15 Salo JTK and Tukiainen EJ: Oncologic resection and reconstruction of the chest wall: A 19-year experience in a single center. Plast Reconstr Surg 142(2): 536-547, 2018. PMID: 29889739. DOI: $10.1097 /$ PRS.0000000000004597

16 Angelini A, Guerra G, Mavrogenis AF, Pala E, Picci P and Ruggieri P: Clinical outcome of central conventional chondrosarcoma. J Surg Oncol 106(8): 929-937, 2012. PMID: 22649023. DOI: $10.1002 /$ jso.23173

17 Mavrogenis AF, Angelini A, Drago G, Merlino B and Ruggieri P: Survival analysis of patients with chondrosarcomas of the pelvis. J Surg Oncol 108(1): 19-27, 2013. PMID: 23681650. DOI: $10.1002 /$ jso.23351

18 Fede C, Stecco C, Angelini A, Fan C, Belluzzi E, Pozzuoli A, Ruggieri $\mathrm{P}$ and De Caro R: Variations in contents of hyaluronan in the peritumoral micro-environment of human chondrosarcoma. J Orthop Res 37(2): 503-509, 2019. PMID: 30444002. DOI: $10.1002 /$ jor. 24176
19 Shiba S, Okamoto M, Kiyohara H, Okano N, Yoshimoto Y, Murata H, Irie D, Katoh $\mathrm{H}$ and Nakano T: Clinical advantage of chest-wall post-mastectomy radiation therapy without bolus. In Vivo 32(4): 961-965, 2018. PMID: 29936486. DOI: 10.21873/ invivo. 11335

20 Weinberg L, Pyo MH, Spanger M and Barnett SA: Personalised 3D-printed model of a chest-wall chondrosarcoma to enhance patient understanding of complex cardiothoracic surgery. BMJ Case Rep, 2018. PMID: 29804079. DOI: 10.1136/bcr-2018224464

21 Ersoz E, Evman S, Alpay L, Akyil M, Vayvada M, Gurer D, Bayram S and Tezel C: Chondrosarcoma of the anterior chest wall: surgical resection and reconstruction with titanium mesh. J Thorac Dis 6(10): E230-233, 2014. PMID: 25364538. DOI: 10.3978/j.issn.2072-1439.2014.09.30

22 Kawana S, Yamamoto H, Maki Y, Sugimoto S, Toyooka S and Miyoshi S: Reconstruction of anterior chest wall with polypropylene mesh: two primary sternal chondrosarcoma cases. Acta Med Okayama 71(3): 259-262, 2017. PMID: 28655947. DOI: $10.18926 / \mathrm{AMO} / 55210$

23 Getz P, Mommsen P, Clausen JD and Winkelmann M: Limited influence of flail chest in patients with blunt thoracic trauma - a matched-pair analysis. In Vivo 33(1): 133-139, 2019. PMID: 30587613. DOI: 10.21873 /invivo. 11449

24 Fiorenza F, Abudu A, Grimer RJ, Carter SR, Tillman RM, Ayoub $\mathrm{K}$, Mangham DC and Davies AM: Risk factors for survival and local control in chondrosarcoma of bone. J Bone Joint Surg $\mathrm{Br}$ 84(1): 93-99, 2002. PMID: 11837841. DOI: 10.1302/0301620x.84b1.11942

25 Lee FY, Mankin HJ, Fondren G, Gebhardt MC, Springfield DS, Rosenberg AE and Jennings LC: Chondrosarcoma of bone: an assessment of outcome. J Bone Joint Surg Am 81(3): 326-338, 1999. PMID: 10199270. DOI: 10.2106/00004623-19990300000004

26 Marulli G, Duranti L, Cardillo G, Luzzi L, Carbone L, Gotti G, Perissinotto E, Rea F and Pastorino U: Primary chest wall chondrosarcomas: results of surgical resection and analysis of prognostic factors. Eur J Cardiothorac Surg 45(6): e194-201, 2014. PMID: 24616390. DOI: 10.1093/ejcts/ezu095

27 Streitbuerger A, Ahrens H, Gosheger G, Henrichs M, Balke M, Dieckmann R and Hardes J: The treatment of locally recurrent chondrosarcoma: Is extensive further surgery justified? J Bone Joint Surg Br 94(1): 122-127, 2012. PMID: 22219259. DOI: 10.1302/0301-620X.94B1.26876

28 Walsh GL, Davis BM, Swisher SG, Vaporciyan AA, Smythe WR, Willis-Merriman K, Roth JA and Putnam JB, Jr: A singleinstitutional, multidisciplinary approach to primary sarcomas involving the chest wall requiring full-thickness resections. J Thorac Cardiovasc Surg 121(1): 48-60, 2001. PMID: 11135159. DOI: $10.1067 / \mathrm{mtc} .2001 .111381$

29 Zheng B, Qu Y, Wang J, Shi Y and Yan W: Pathogenic and targetable genetic alterations in resected recurrent undifferentiated pleomorphic sarcomas identified by targeted next-generation sequencing. Cancer Genomics Proteomics 16(3): 221-228, 2019. PMID: 31018952. DOI: 10.21873/cgp.20127

Received January 7, 2020

Revised January 27, 2020

Accepted February 4, 2020 